CASE SERIES

\title{
Full-mouth Rehabilitation of Worn Dentition by Hobo Twin-stage Philosophy: A Case Series
}

\author{
Huzaifa Ezzy ${ }^{1}$, Amit Gaikwad ${ }^{2}$, Sabita Ram ${ }^{3}$, Naisargi Shah ${ }^{4}$, Jyoti Nadgere ${ }^{5}$, Janani lyer ${ }^{6}$
}

\begin{abstract}
Esthetic and functional restoration of the severely worn dentition represents a significant clinical challenge to the dentist. Diagnosis is most important in treatment planning. A systematic, phase wise approach is of utmost importance in full-mouth rehabilitation. Over time, various concepts and philosophies to attain reconstruction and rehabilitation of the entire dentition have been proposed. This article focuses on the phase-by-phase treatment of two patients with worn dentition by the Hobo Twin-stage procedure.
\end{abstract}

Keywords: Full-mouth rehabilitation, Hobo Twin-stage, Tooth wear.

Journal of Contemporary Dentistry (2019): 10.5005/jp-journals-10031-1246

\section{INTRODUCTION}

Full-mouth rehabilitation of worn out or attrited dentition involves the development of sufficient restorative space, while simultaneously fulfilling dentofacial, esthetic, functional, periodontal, and occlusal parameters essential for long-term success. Tooth wear is a threat to the dentition and masticatory function. Various factors may combine to produce worn out dentition and the etiology often remains unidentified. ${ }^{1}$ Tooth wear may be due to tooth contact, clenching, mechanical action of external agents, chemical erosion, or noncarious cervical abfraction. ${ }^{2,3}$ It can occur without any signs and symptoms, but may become destructive when increased tooth wear and pain is present. ${ }^{4}$ Severe or excessive wear refers to tooth destruction that requires restorative intervention. Restoration of such dentition is one of the most challenging treatments in dentistry. Rehabilitation of occlusal form and function becomes the primary objective in such cases and they often require a fullmouth rehabilitation. Various popular occlusal philosophies and concepts can be followed in full-mouth rehabilitations such as Gnathological concept, Pankey-Mann-Schuyler concept, Hobo concept, Biological occlusion concept, Youdelis concept, and Nyman and Lindhe concept. ${ }^{5}$

\section{Clinical Report}

A 72-year-old male patient reported at the Department of Prosthodontics with the chief complaint of loss of tooth structure in his dentition. The patient was concerned with his appearance and chewing ability. The patient was in good general health, and the medical and dental history indicated no contraindications for dental treatment. On extraoral examination, the patient had an ovoid facial form, convex profile, uneven occlusal plane, competent lips, and inadequate lip support. Smile line did not coincide with the lips. The patient revealed an average smile line with no gingival display. No signs and symptoms were found in the temporomandibular joints. The vertical dimension appeared over closed. Prominent masseter muscle activity was present. On intraoral examination, the oral hygiene was average with no symptoms of periodontal affliction. On intraoral examination, generalized attrition/wear and abrasion was present, discolored teeth, and carious 16 was observed. Periodontal examination revealed grade II mobility with respect $\overline{1-6}$ Department of Prosthodontics, MGM Dental College and Hospital, Navi Mumbai, Maharashtra, India

Corresponding Author: Huzaifa Ezzy, Department of Prosthodontics, MGM Dental College and Hospital, Navi Mumbai, Maharashtra, India, Phone: +91 8879372027, e-mail: drhuzaifaezzy@gmail.com

How to cite this article: Ezzy H, Gaikwad A, et al. Full-mouth Rehabilitation of Worn Dentition by Hobo Twin-stage Philosophy: A Case Series. J Contemp Dent 2019;9(1):17-24.

Source of support: Nil

Conflict of interest: None

to 31 and 41, furcation involvement and mobility with respect to 47 , and the recession was present with respect to 43 . Existing composite restorations with respect to 11,21 , and 47 , and shiny Amalgam restoration with respect to 46 were observed (Figs 1 to 3 ).

\section{DIAGNOSIS}

In the Turner and Missirlian classification of occlusal vertical dimension and tooth wear, the patient fell in class I-excessive wear with loss of vertical dimension with space available to restore the vertical height.

\section{TREATMENT}

A multiphase treatment protocol was planned as follows:

\section{Phase I: Patient Counseling}

The initial phase began with patient education and motivation about the cause of his present oral condition and treatment plan for the same. Psychological counseling was done. A comprehensive oral evaluation was done and pretreatment records were gathered.

\section{Phase 2: Disease Control Phase}

Periodontal prophylaxis, maintenance, and oral hygiene instructions were given to the patient. A caries management protocol was administered. The patient was asked to switch to soft-bristled toothbrush due to the abrasion present. 


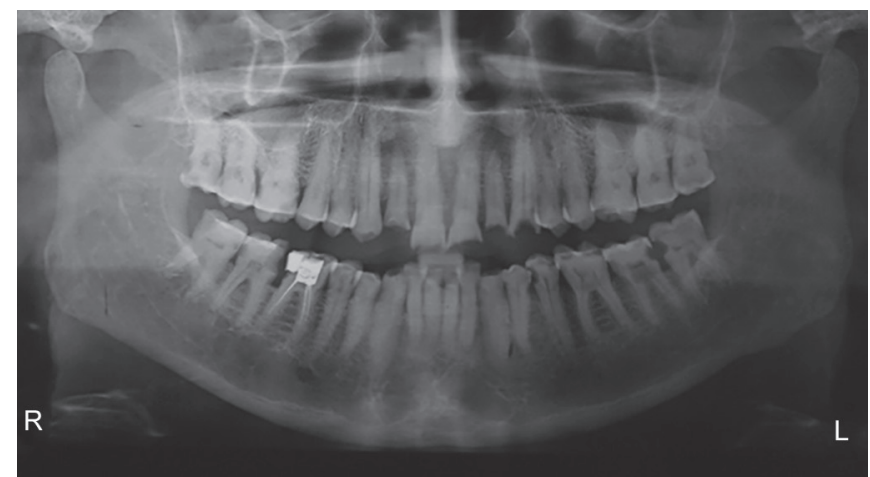

Fig. 1: Orthopantograph (OPG)

\section{Phase 3: Surgical Phase}

Extraction of 31, 41, and 47 was done due to poor prognosis. The extraction sites were allowed to heal for 3 months before restorative therapy commenced.

\section{Phase 4: Diagnostic Procedures}

The vertical dimension was assessed clinically. Physiologic rest position was determined by facial measurements and confirmed by phonetics. The interocclusal distance was judged to be approximately $5 \mathrm{~mm}$, and the occlusal vertical dimension (OVD) could be restored by increasing it approximately $2 \mathrm{~mm}$. Prior to definitive treatment, diagnostic casts were obtained from primary impressions (Alginate, Tropicalgin, Zhermack). The bite registration procedure was accomplished using a customized anterior programming device (impression compound) in the anterior region and interocclusal bite registration polyvinyl siloxane (PVS) material

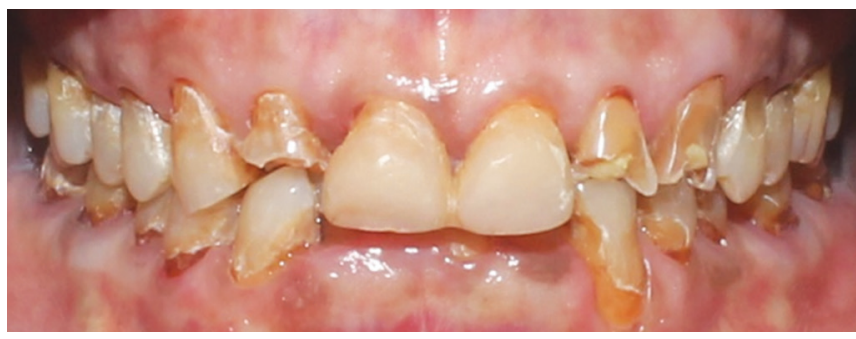

Fig. 2: Intraoral view

injected into the posterior region when the mandible was guided into centric relation (CR) by the bimanual manipulation technique (Fig. 4). Using this record and an arbitrary facebow (Spring-bow; Teledyne, USA) will be recorded and maxillary casts mounted on a semiadjustable articulator the casts were mounted on a semiadjustable articulator (HANAU articulator mode Wide-Vue, USA). The incisal pin was adjusted for a 2-mm opening and wax-up was done following the Hobo Twin-stage philosophy.

\section{Hobo Twin-stage Procedure}

The wax-up was done on the semiadjustable articulator in two stages (conditions). During the waxing of the occlusal morphology under condition I, the anterior portion of the working cast became an obstacle because it produced disocclusion during eccentric movement. Therefore, when the posterior wax-up was made, the anterior segment of the working cast was made by using removable dowel pins. Posterior wax patterns were fabricated such that there are smooth gliding contacts from CR to protrusive and lateral movements (Fig. 5). This would ensure a uniform amount
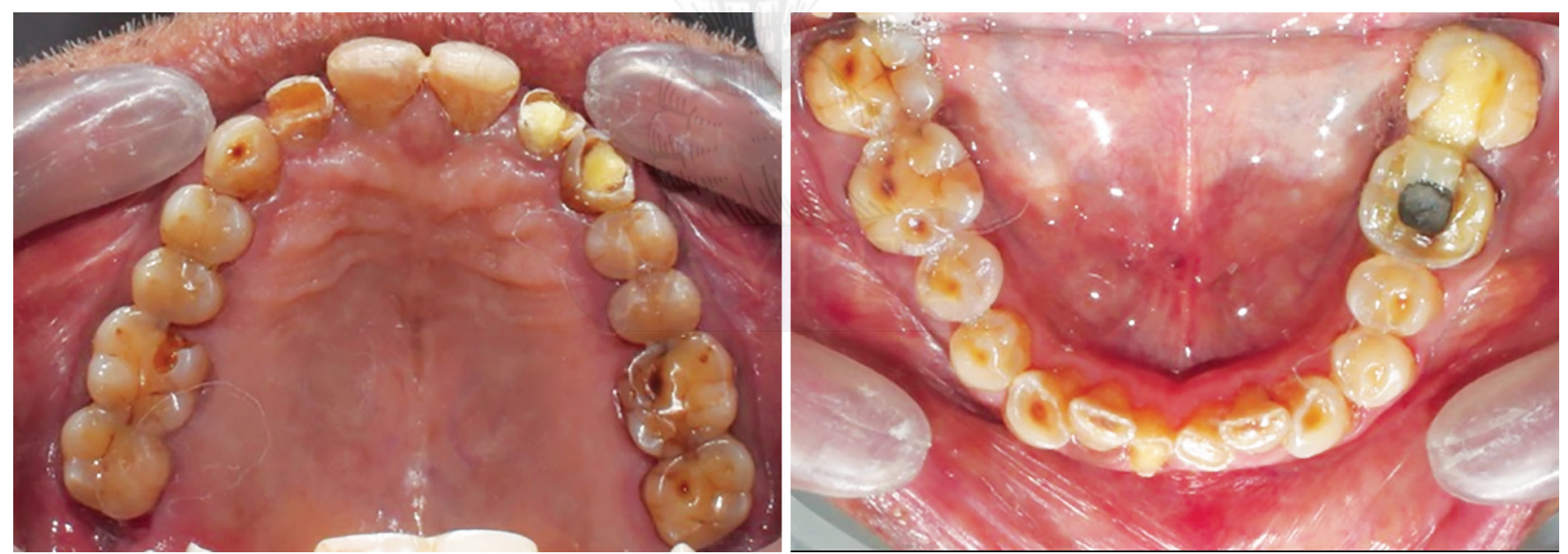

Fig. 3: Intraoral maxillary and mandibular view
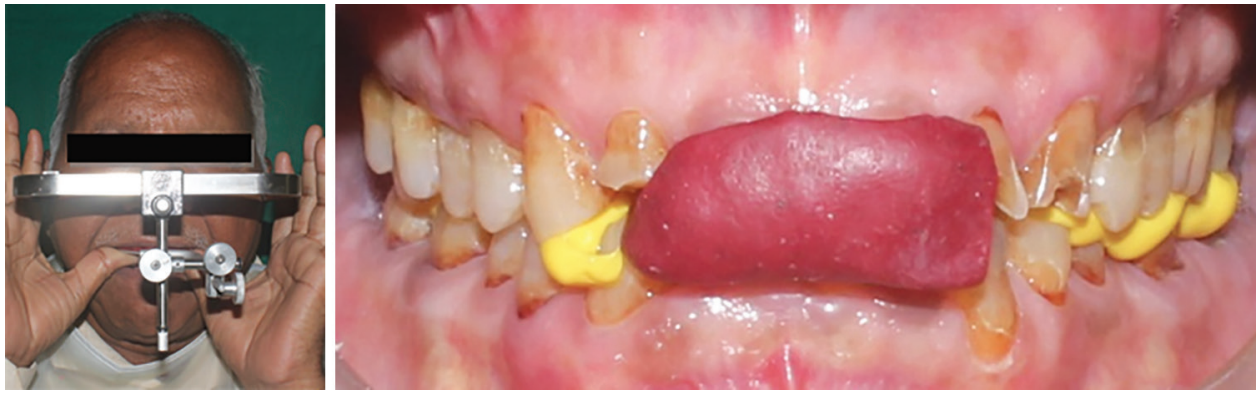

Fig. 4: Facebow bite registration with lucia jig 

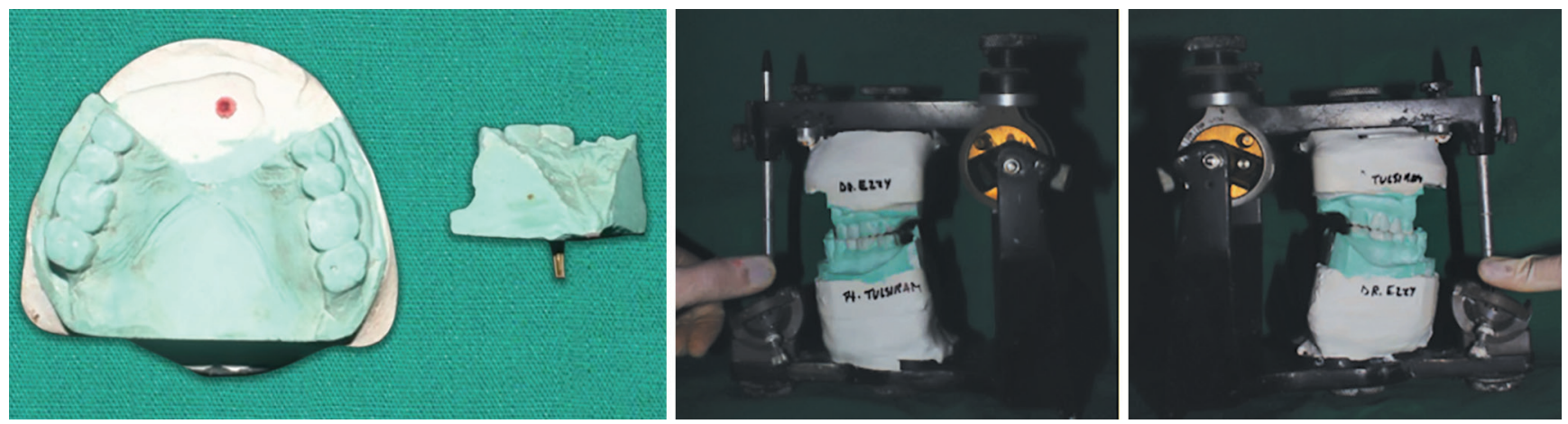

Fig. 5: Condition I-anterior segment removed and posterior balanced

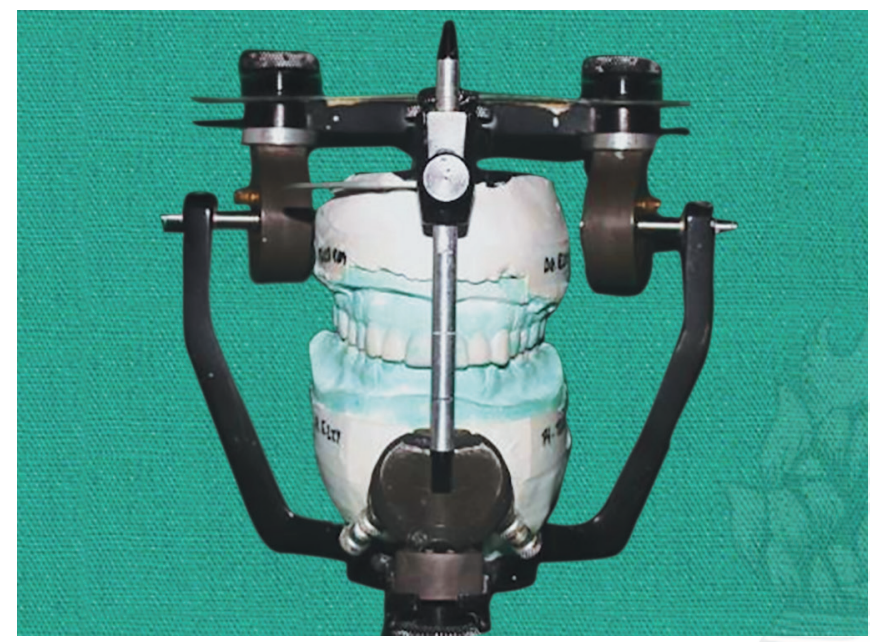

Fig. 6: Condition II

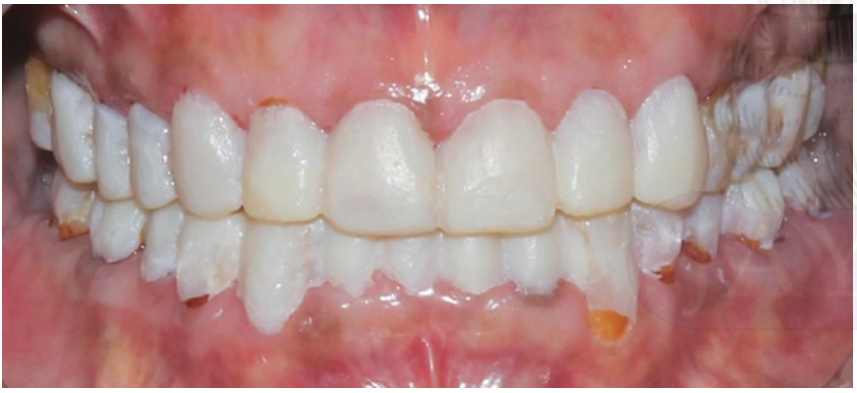

Fig. 7: Trial of wax-up

of posterior disocclusion during lateral and protrusive excursions when the anterior guidance is established later. The standard cusp angle $\left(25^{\circ}\right)$ was created under condition I on the cast without the anterior segment. It is recommended that the condylar path be adjusted to $40^{\circ}$ and the anterior guide table be adjusted to $45^{\circ}$ to fabricate the anterior guidance. This was the adjustment value for the articulator to achieve condition II. The anterior segment of the removable die system is replaced with the cast and wax patterns were fabricated with the articulator settings. Anterior dies were replaced onto the casts and wax-up is completed to achieve adequate aesthetics (Fig. 6). The palatal contours were adjusted according to the anterior guidance to provide immediate disocclusion away from CR. Adjustment values for the establishment of mutually protected articulation are shown in Table 1.

The Putty Index was then made and bisacryl composite (Protemp 4) and a trial of the wax-up followed by Hobo's philosophy
Table 1: Articulator settings in the Hobo Twin-stage procedurel

\begin{tabular}{|c|c|c|c|c|}
\hline \multirow[b]{2}{*}{ Condition } & \multicolumn{2}{|c|}{ Condylor path } & \multicolumn{2}{|c|}{ Anterior guide table } \\
\hline & $\begin{array}{l}\text { Sagittal } \\
\text { condylar path } \\
\text { inclination }\end{array}$ & $\begin{array}{l}\text { Bennett } \\
\text { angle }\end{array}$ & $\begin{array}{l}\text { Sagittal } \\
\text { inclination }\end{array}$ & $\begin{array}{l}\text { Lateral } \\
\text { wing } \\
\text { angle }\end{array}$ \\
\hline $\begin{array}{l}\text { Condition 1: } \\
\text { Without anterior } \\
\text { teeth }\end{array}$ & 25 & 15 & 25 & 10 \\
\hline $\begin{array}{l}\text { Condition 2: } \\
\text { Without anterior } \\
\text { teeth }\end{array}$ & 40 & 15 & 45 & 20 \\
\hline
\end{tabular}

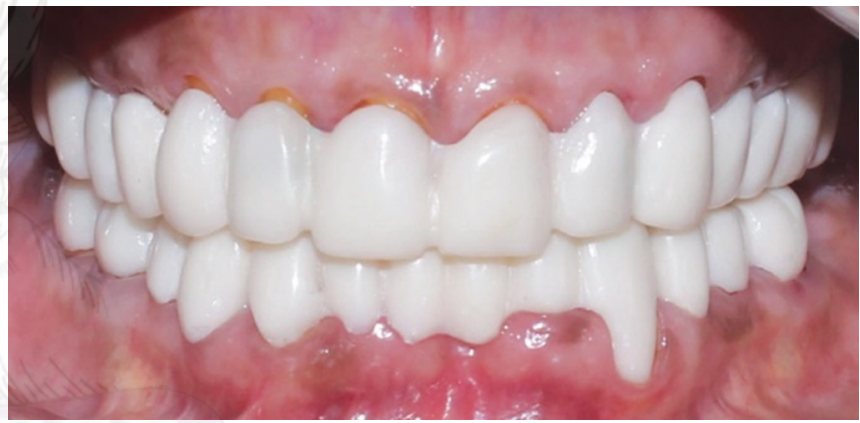

Fig. 8: Temporization

were done intraorally at this new vertical dimension (VD) for evaluation. Esthetics and phonetics were checked. Changes were made intraorally. Centric contacts were evaluated and adjusted and disocclusion was checked in eccentric movements (Fig. 7).

\section{Phase 5: Preprosthetic Procedures}

With the aid of the above diagnostic procedure, we could get a clear idea for our definitive prosthesis and, hence, a definite treatment plan was followed. The preprosthetic procedures required for the patient were then completed having our approved diagnostic wax-ups as our guide. They were as follows:

- Root canal treatment of 12, 22, 23, 32, 33, 42, and 43

- Cast post with 12,22 , and 23

- I post with 32, 33, 42, and 43

- Composite restoration with 16,26 , and 27

\section{Phase 6: Temporization}

Once it was approved, an alginate impression was made as a reference. Gross preparation was done and heat cure provisional crowns were fabricated for the same (Fig. 8). The patient used these prostheses for 3 months to check the proposed vertical dimension. 


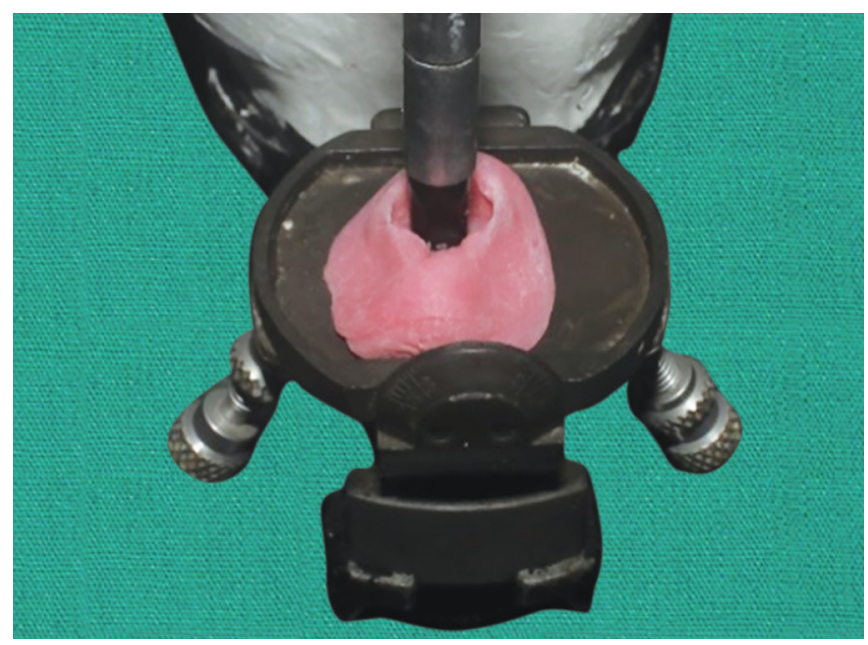

Fig. 9: Customized anterior guide table

After 3 months, the patient was satisfied with this new OVD without any signs and/or symptoms. The patient was now with a functionally guided temporary prosthesis which was followed by the Hobo Twin-stage philosophy. A second series of diagnostic casts obtained from impressions (Alginate) was mounted on a semiadjustable articulator (Hanau) using an interocclusal registration in CR when the mandible was guided into CR using the bimanual manipulation technique as mentioned before. A customized anterior guide table was made (Fig. 9).

\section{Phase 7: Tooth Preparations, Impressions, Jaw Relation Record (Figs 10 and 11)}

Final tooth preparation was done in the maxillary arch using a thermoplastic sheet made from the above impression as a guiding stent. The final impression was made in putty and light body with the two-stage technique. The facebow record was made and the interocclusal record was taken. The temporary crowns aided in recording at the desired correct vertical dimension and a lucia jig (impression compound) was fabricated, interocclusal record in the posterior region was done by Alu wax. Records, casts, and final impressions were transferred to the articulator for the fabrication of definitive prosthesis. The metal trial was done and bisque trial of single porcelain fused to metal (PFM) crowns was done in the maxillary arch. The shade finalized was A2 with characterization. The shape and the form of teeth were selected keeping in mind
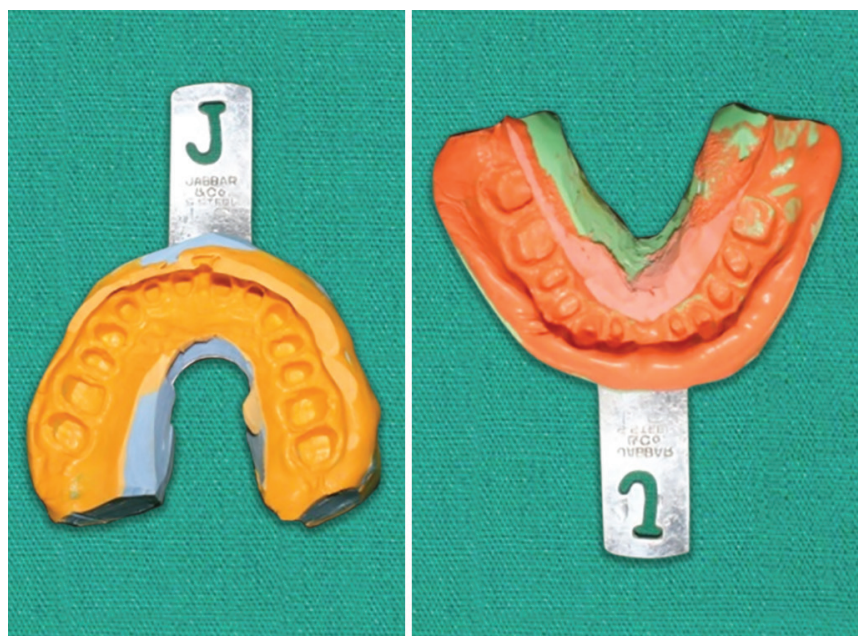

Fig. 11: Maxillary and mandibular final impressions

the sex age personality (SPA) factor. The maxillary anterior crowns, thus, fabricated were cemented with temporary luting cement and evaluated for esthetics, functional harmony, and comfort.

The same was repeated for the mandibular arch. It consisted of a 6 unit PFM bridge in the mandibular anterior region, single crowns in the right posterior region, and 4 unit bridge with the cantilever in the left posterior region. The mandibular prosthesis was fabricated following the contours of the maxillary incisal and occlusal surfaces on the articulator. Bisque trial of maxillary and mandibular teeth was done and temporarily cemented to check the occlusion and guidance. Digital occlusal analysis and adjustment of the definitive prosthesis were done with the help of T-scan (Fig. 12). Cementation of the definitive prosthesis was done with the zinc phosphate cement (Figs 13 and 14).

\section{Phase 8: Maintenance Phase}

A maxillary night guard was fabricated (thermoplastic sheet). The patient was scheduled to return for postinsertion recalls and instructed to adhere to his daily nightguard regimen (Fig. 15).

\section{Case 2-Minimally Invasive with Bonded Restorations}

A 54-year-old female patient reported at the Department of Prosthodontics with the chief complaint of sensitivity and small
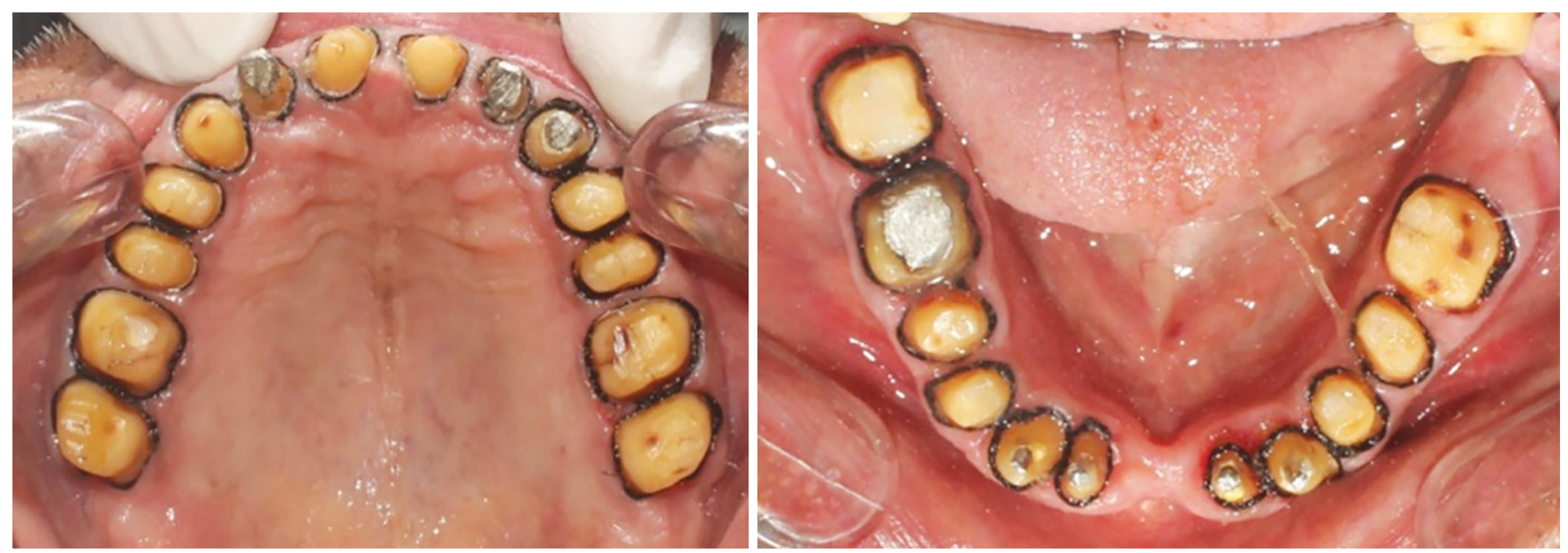

Fig. 10: Maxillary and mandibular preparation 

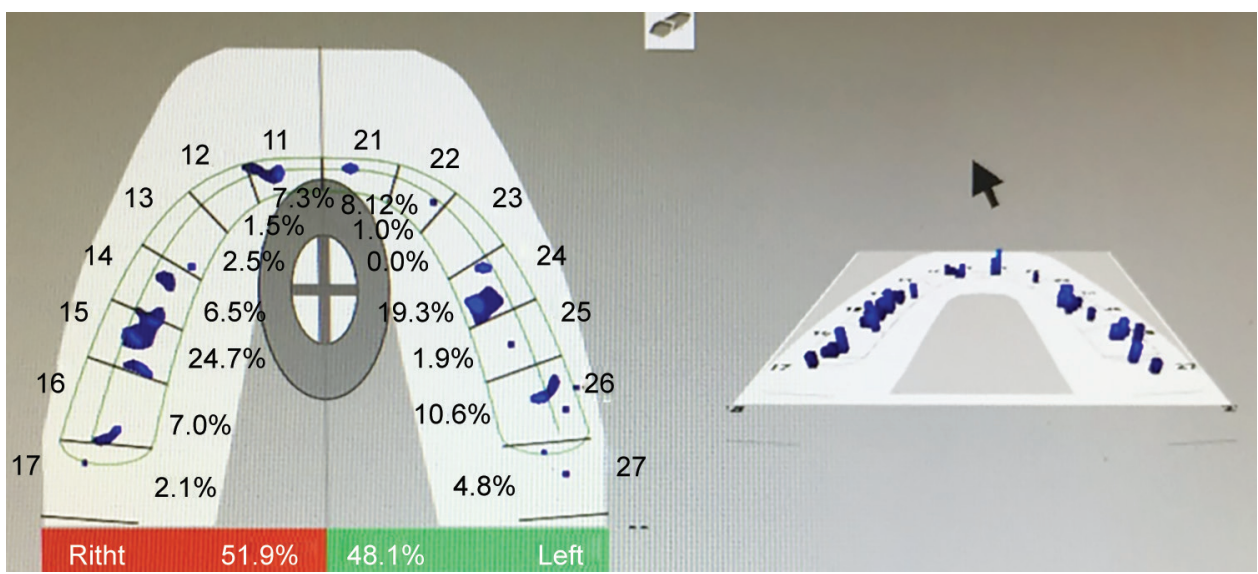

Fig. 12: T-scan analysis
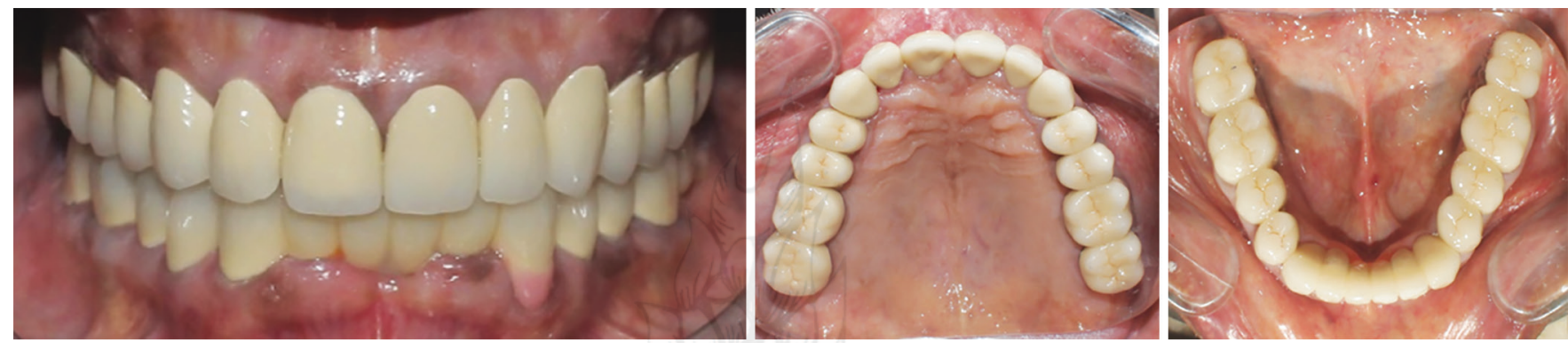

Fig. 13: Postoperative intraoral view
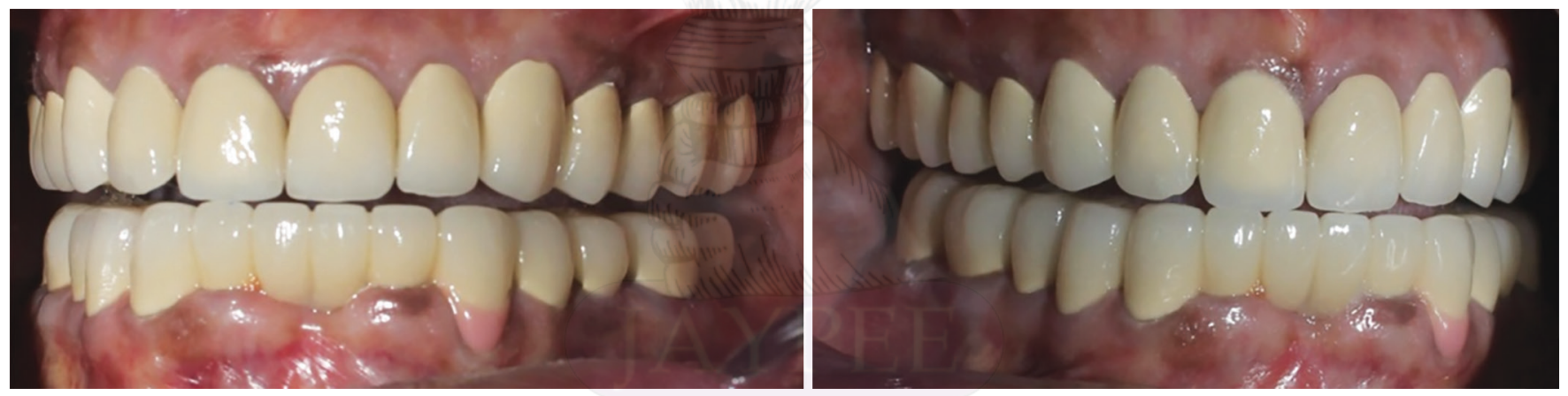

Fig. 14: Uniform posterior disocclusion achieved

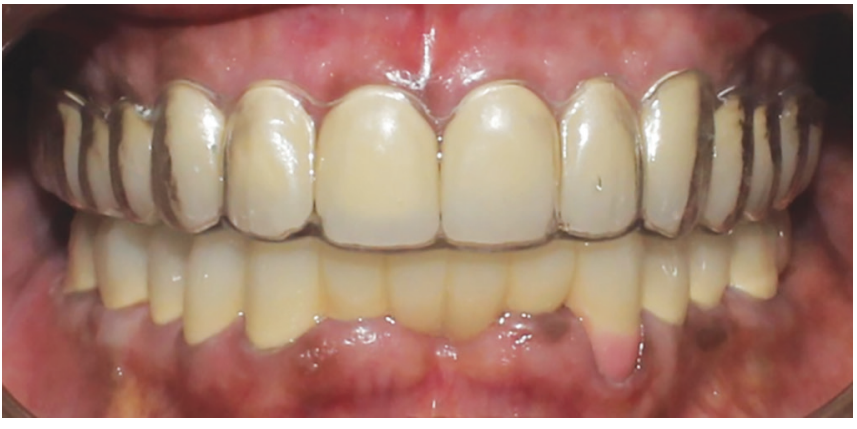

Fig. 15: Maxillary night guard as maintenance

upper and lower front teeth since 1 year. The patient gave a history of pain in the morning near the temporomandibular joint and with a moderate headache. Slight hypertrophy was observed with the masseter muscle with no signs of pain and inflammation. On smiling, $100 \%$ of teeth were visible with $25 \%$ of gingival display.
There was loss of incisal embrasure giving a flat appearance of the upper occlusal plane.

Intraoral examination showed wear on the incisal and occlusal surface of the upper and lower anterior and posterior teeth. The available crown height for 31, 32, 41, and 42 was $3.6 \mathrm{~mm}$ and, for 33 and 43 , it was $4.8 \mathrm{~mm}$. The patient had group function occlusion on the right and the left side. Other history was not relevant (Fig. 16).

Radiographic examination showed an adequate bone height in the maxillary and the mandibular region. Periodontal ligament widening was observed in the mandibular anterior region. The Niswonger method of vertical dimensions showed on an average of $5 \mathrm{~mm}$ of available space for restoration and diagnostic mounting was done on a semiadjustable articulator. Radiographic analysis and diagnostic cast mounted on an articulator suggested turner class II situation. After explaining various treatment options, minimal invasive with laminate for anterior teeth and onlays for posterior teeth using the Hobo Twin-stage technique was the final treatment plan decided and agreed by the patient. Diagnostic wax-up was 

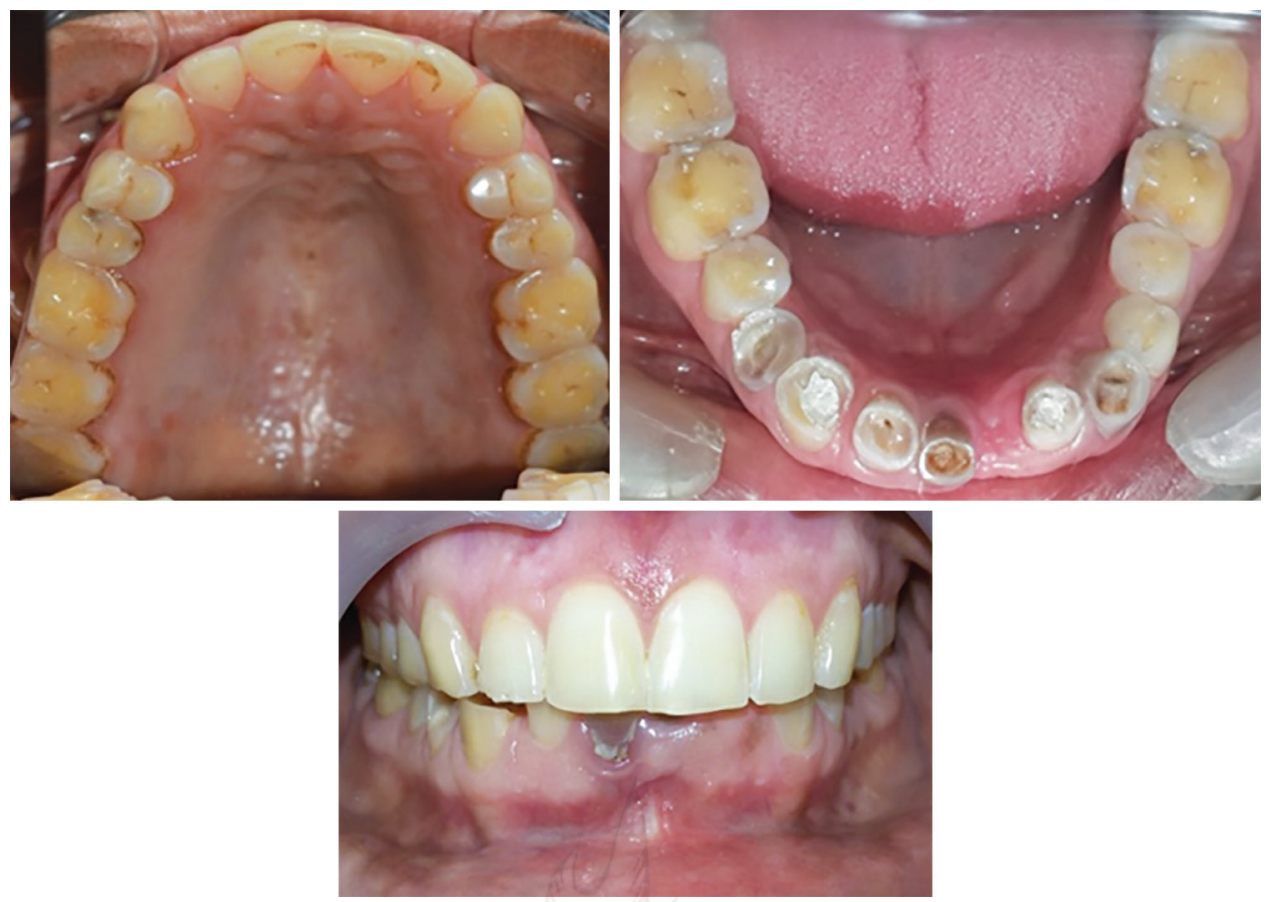

Fig. 16: Preoperative intraoral view

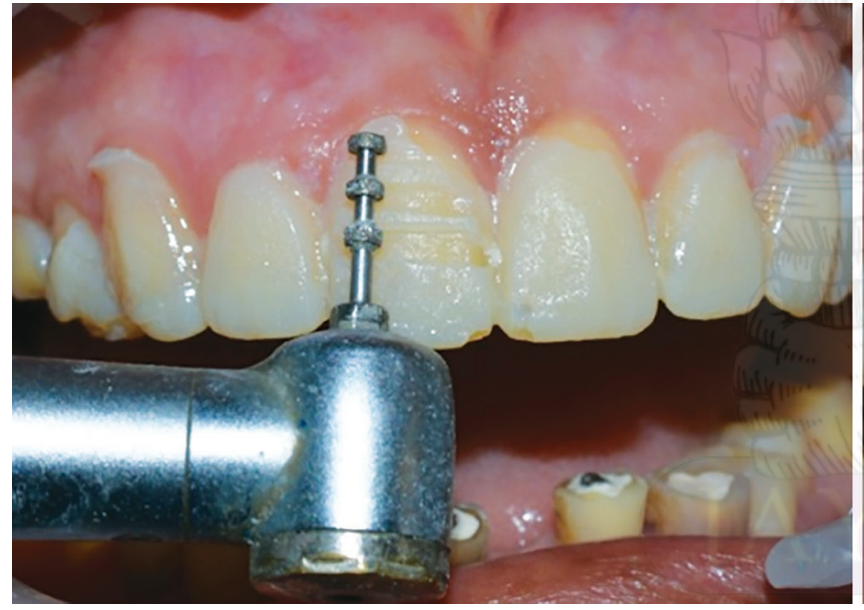

Fig. 17: Minimal invasive tooth preparation

done using the Hobo Twin-stage technique. Occlusal plane and esthetic were evaluated intraorally using the Putty Index and Protemp 4. Tooth preparation of all teeth was done with depth cutting burs and using the Putty Index which helped in achieving minimal tooth reduction (Fig. 17). Occlusal reduction of posterior teeth was avoided as the interocclusal space available was adequate for restoration. Provisional restoration of all teeth was done with Protemp 4 using the Putty Index. The patient was evaluated for esthetic, phonetic, and comfort of the patient. The patient was kept on recall until 3 months.

After 3 months, the patient was again evaluated for esthetic, phonetics, comfort, and function. Maxillary and mandibular final impressions were done by adding silicone after gingival retraction. The mandibular anterior segment was sectioned and removed. The maxillary master cast was mounted using facebow record and the lower cast was mounted using the interocclusal record. Final teeth wax-up was done using the Hobo Twin-stage technique.

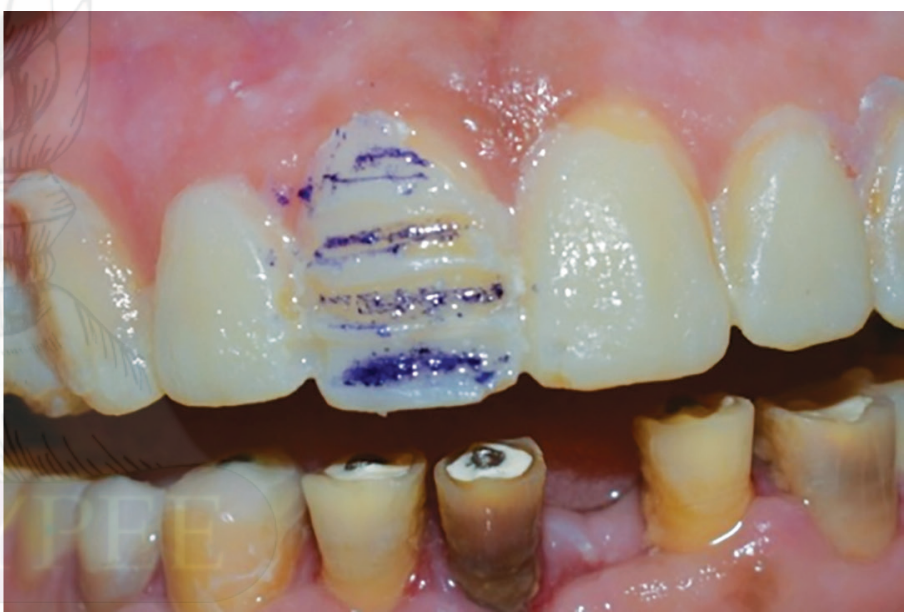

For bonding of lithium disilicate, first etching of teeth was done with $37 \%$ orthophosphoric acid for 10 seconds. Simultaneously, the restorations were etched with $10 \%$ hydrofluoric acid. The etched surface of both teeth and restoration showed white frosted appearance. Teeth were isolated and air dried and the fourth generation bonding agent was applied. Simultaneously, a silane coupling agent was applied on restoration. Final bonding of all restorations was done using the light cure resin cement (Fig. 18). Occlusion was evaluated using a 40- and 12-micron Bausch articulating paper. Night guard was fabricated for the lower arch and delivered to the patient. Recall of the patient was done after 24 hours, 3 days, 3 months, and every 6 months (Figs 19 and 20).

\section{Discussion}

Early gnathological concepts focused primarily on the condylar path as it was theorized to be a constant through adulthood. Anterior guidance was considered to be at the discretion of the 

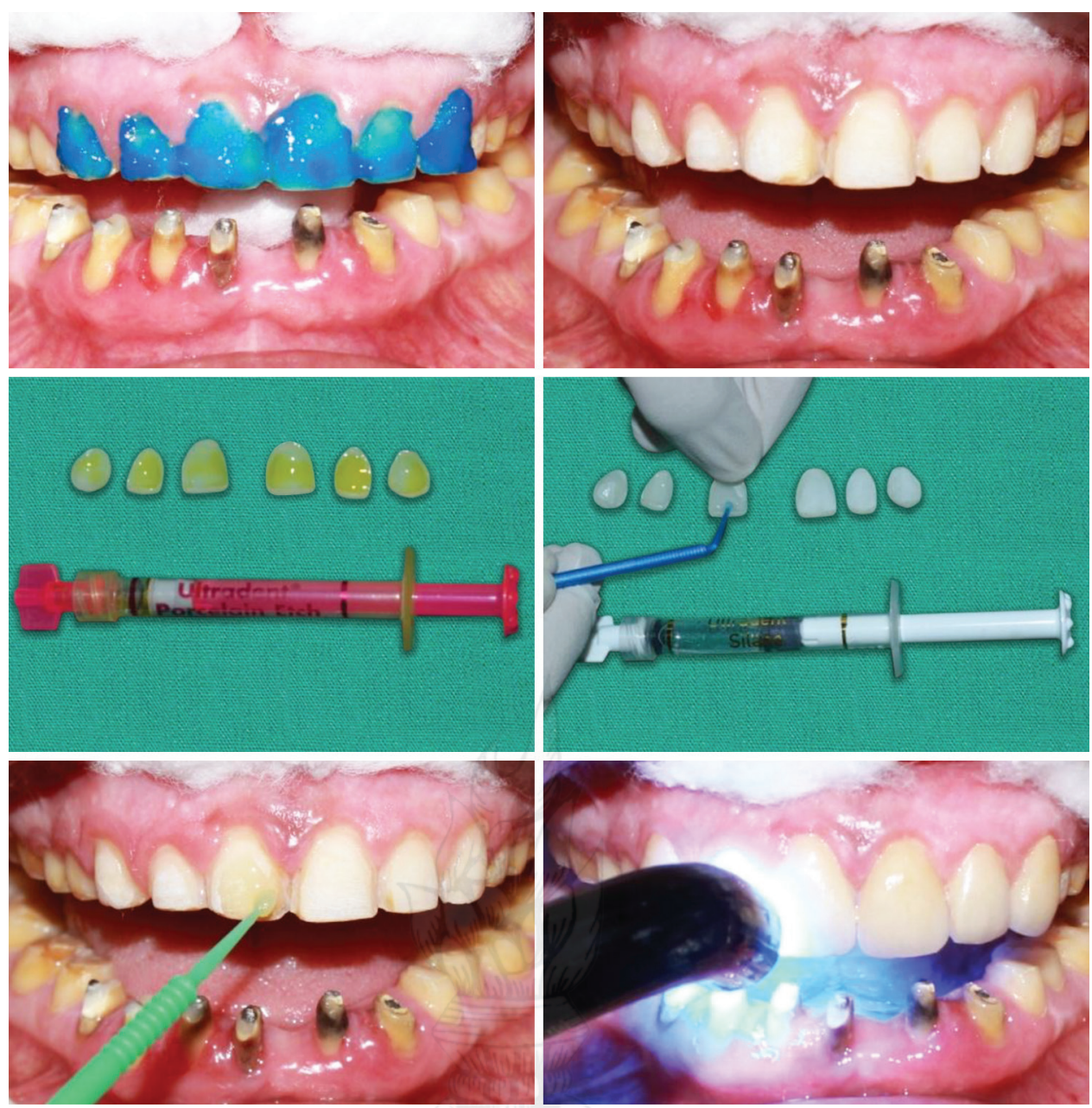

Fig. 18: Bonding protocol
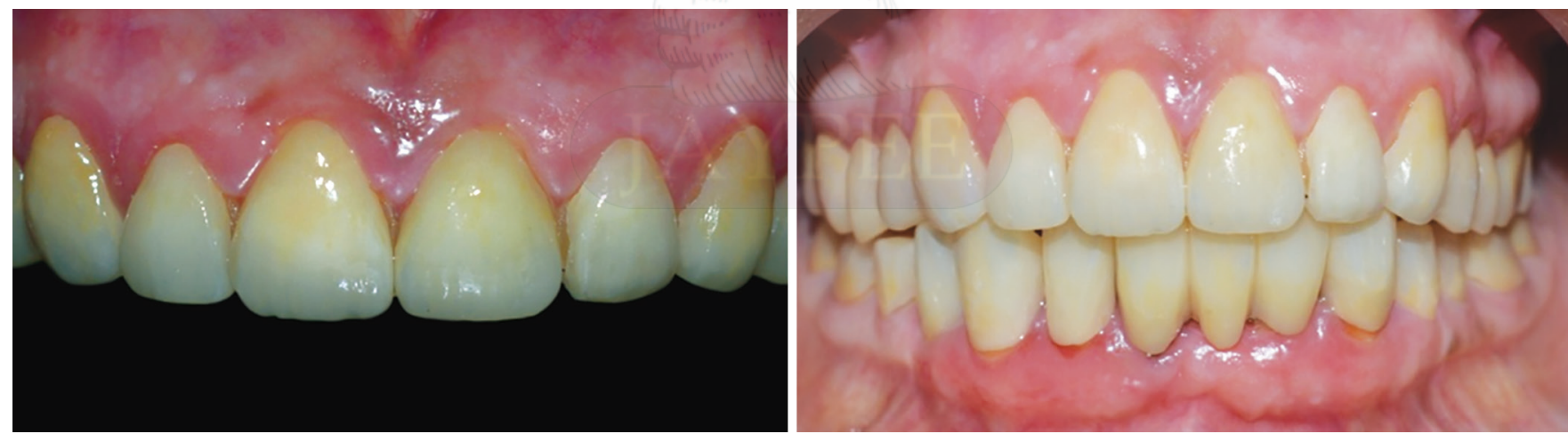

Fig. 19: Intraoral postoperative

dentist. McCollum and Stuart concluded from a study conducted on 10 patients that condylar guidance is dependent on the anterior guidance. ${ }^{6}$ In prosthodontics, the condylar path has been considered the main determinant of occlusion. According to the twin-table technique by Hobo, the cusp shape factor and the angle of hinge rotation are derived from the condylar path. ${ }^{6}$ These factors contribute to the determination of an ideal anterior guidance. However, in the twin-stage procedure, the cusp angle was considered as the most reliable determinant of occlusion. This was according to the proven data from studies that the cusp angle was four times more reliable than condylar and incisal paths. ${ }^{7}$

In the twin-stage procedure, to provide disocclusion, the cusp angle should be shallower than the condylar path. To make a shallower cusp angle in a prosthesis, it is important to wax the occlusal morphology to produce balanced occlusion or articulation so that the cusp angle becomes parallel to the cusp path of opposing teeth during eccentric movements. ${ }^{8}$ Since anterior teeth help to produce disocclusion, when a dentist waxes the occlusal morphology and tries to reproduce a shallower cusp angle, the 


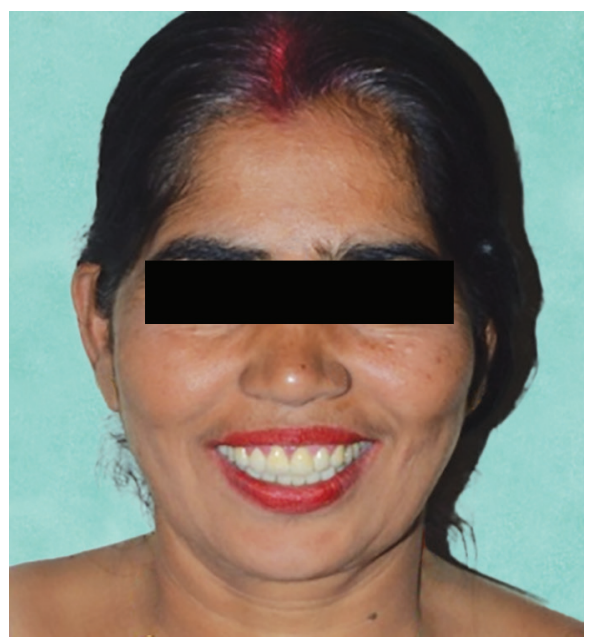

Fig. 20: Extraoral postoperative

anterior portion of the working cast becomes an obstacle. Also, when fabricating the anterior teeth to produce disocclusion, some guidance should be incorporated. In this conditional approach described by Hobo, a cast with a removable anterior segment is fabricated. Reproduction of the occlusal morphology of the posterior teeth is done without the anterior segment and a cusp angle coincident with the standard values of effective cusp angle is produced (referred to as "condition I"). ${ }^{9}$ Second, reproduction of the anterior morphology with the anterior segment is done and anterior guidance which produces a standard amount of disocclusion is provided (referred to as "condition II"). ${ }^{9}$

Since the measurement of the condylar path is not necessary, complicated instruments such as the pantograph and fully adjustable articulator become unnecessary. Therefore, the twinstage procedure is much simpler than the standard gnathological procedure, yet it follows gnathological principles. Since the condylar path is not used as the main determinant of occlusion, this procedure is suitable for restorative work for patients with temporomandibular disorders and splint therapy. ${ }^{5}$ This procedure can be incorporated easily with commonly used clinical techniques such as facebow transfer, various centric recording methods, and cusp-fossa waxing. The contraindications of this technique are abnormal curve of Spee, abnormal curve of Wilson, abnormally rotated teeth, and abnormally inclined teeth ${ }^{5}$.

\section{Conclusion}

By understanding the individual risk factors both esthetically and functionally, a treatment plan was designed to minimize any additional risk to the remaining dentition. Correct diagnosis and a systematic, phase wise approach are of utmost importance in full-mouth rehabilitation. The maintenance of severe wear cases can be ensured by the development of proper cuspal angulation and anterior guidance that allows for posterior disocclusion within the patient's envelope of function. Taking this guidance into account during temporization ensures minimal adjustments in the definitive restorations and a greater long-term predictability and better prognosis of the case.

\section{References}

1. Crothers AJ. Tooth wear and facial morphology. J Dent 1992 Dec 1;20(6):333-341. DOI: 10.1016/0300-5712(92)90019-9.

2. Grippo JO, Simring M, et al. Attrition, abrasion, corrosion and abfraction revisited: a new perspective on tooth surface lesions. J Am Dent Assoc 2004 Aug 1;135(8):1109-1118. DOI: 10.14219/jada.archive.2004.0369.

3. Verrett RG. Analyzing the etiology of an extremely worn dentition. J Prosthodont 2001 Dec;10(4):224-233. DOI: 10.1111/j.1532849X.2001.00224.x.

4. Van't Spijker A, Kreulen CM, et al. Attrition, occlusion, (dys) function, and intervention: a systematic review. Clin Oral Implants Res 2007 Jun;18:117-126. DOI: 10.1111/j.1600-0501.2007.01458.x.

5. Shetty BR, Shetty M, et al. Philosophies In Full Mouth RehabilitationA Systematic Review. Int J Dent Case Rep 2013;3:30-39.

6. Hobo S. Twin-tables technique for occlusal rehabilitation: part I-mechanism of anterior guidance. J Prosthet Dent 1991 Sep 1;66(3):299-303. DOI: 10.1016/0022-3913(91)90253-S.

7. Hobo S. Twin-tables technique for occlusal rehabilitation: part IIClinical procedures. J Prosthet Dent 1991 Oct 1;66(4):471-477. DOI: 10.1016/0022-3913(91)90507-S.

8. Hobo S, Takayama H. Oral rehabilitation: clinical determination of occlusion. Quintessence Pub Co 1997.

9. Hobo S, Takayama H. Twin-stage procedure. Part 1: a new method to reproduce precise eccentric occlusal relations. Int J Periodontics Restorative Dent 1997 Apr 1;17(2):112-123. 